\title{
A case of aquagenic syringeal acrokeratoderma with involvement of periungual area of the hand
}

\author{
Gülhan Gürel ${ }^{1}$, Sevinç Şahin², Emine Çölgeçen ${ }^{1}$ \\ Departments of ${ }^{1}$ Dermatology and ${ }^{2}$ Pathology, Bozok University Faculty of Medicine, Yozgat, Turkey. \\ E-mail: gulhanozturkgurel@hotmail.com
}

Received: 6th January 2017, Revised: 11th November 2017, 11th December 2017, Accepted: 24th December 2017

\begin{abstract}
SUMMARY: Gürel G, Şahin S, Çölgeçen E. A case of aquagenic syringeal acrokeratoderma with involvement of periungual area of the hand. Turk J Pediatr 2018; 60: 762-764.

Aquagenic syringeal acrokeratoderma (ASA) is a rare form of palmoplantar keratoderma occurring after short-term contact with water. Although ASA usually involves the palmar region, there are also several cases with the involvement of dorsum of hand and sole of the feet. We described 15-year old girl who had white keratodermic plaques observed on the flexor side of distal phalanxes especially the periungual area after a 10 min contact with water. Our patient represents a rare case of ASA with the involvement of periungual region of the fingers.
\end{abstract}

Key words: aquagenic syringeal acrokeratoderma, adolescent, water.

Aquagenic syringeal acrokeratoderma (ASA) is a rare form of palmoplantar keratoderma occurring after short-term contact with water. ${ }^{1}$ It was first defined in 1996 by English and McCollough ${ }^{1,2}$ in the name of 'transient reactive papulotranslucent acrokeratoderma' and then called 'aquagenic palmoplantar keratoderma' and 'aquagenic syringeal acrokeratoderma' by other authors.

\section{Case Report}

A 15-year old female patient was admitted to clinic with the complaint of blistering for about 1 year after water exposure which began on the palm of the hand and spread around the fingers. The patient reported that her complaints increase with water contact for $10 \mathrm{~min}$ and alleviate with a $20 \mathrm{~min}$ resting period after drying the hands. The patient also stated that she had excessive sweating on her hands. After a 10 min contact with water, white keratodermic plaques were observed on the flexor side of the distal phalanxes and especially periungual area (Fig. 1-2). Lesions completely disappeared after about $20 \mathrm{~min}$ following drying the hands. She had palmar hyperhidrosis. Neither the patient nor her family had history of cystic fibrosis. Punch biopsy was performed from the periungual area of the left third finger of the patient.

Histopathological examination of the lesions showed orthokeratotic hyperkeratosis on the surface with the dilatation of acrosyringium and a tortuous appearance. Slightly evident irregular acanthosis was found in the epidermis with slight perivascular lymphocytic infiltration in the superficial dermis (Fig. 3 A-D).

In light of the above-mentioned clinical and histopathological findings, a diagnosis of ASA was made and topical $20 \%$ aluminum chloride hexahydrate was prescribed. Partial response was observed after 1-month of treatment. Written informed consent was obtained from the parents of the patient.

\section{Discussion}

ASA is characterized by localized, transparent and white-colored papules and plaques commonly in the palmar region of hand.13 Although it is commonly asymptomatic, patients may complain from burning, pain, itching, and tingling. Hyperhidrosis may accompany the active disease. ${ }^{4}$

Although ASA usually involves the palmar 


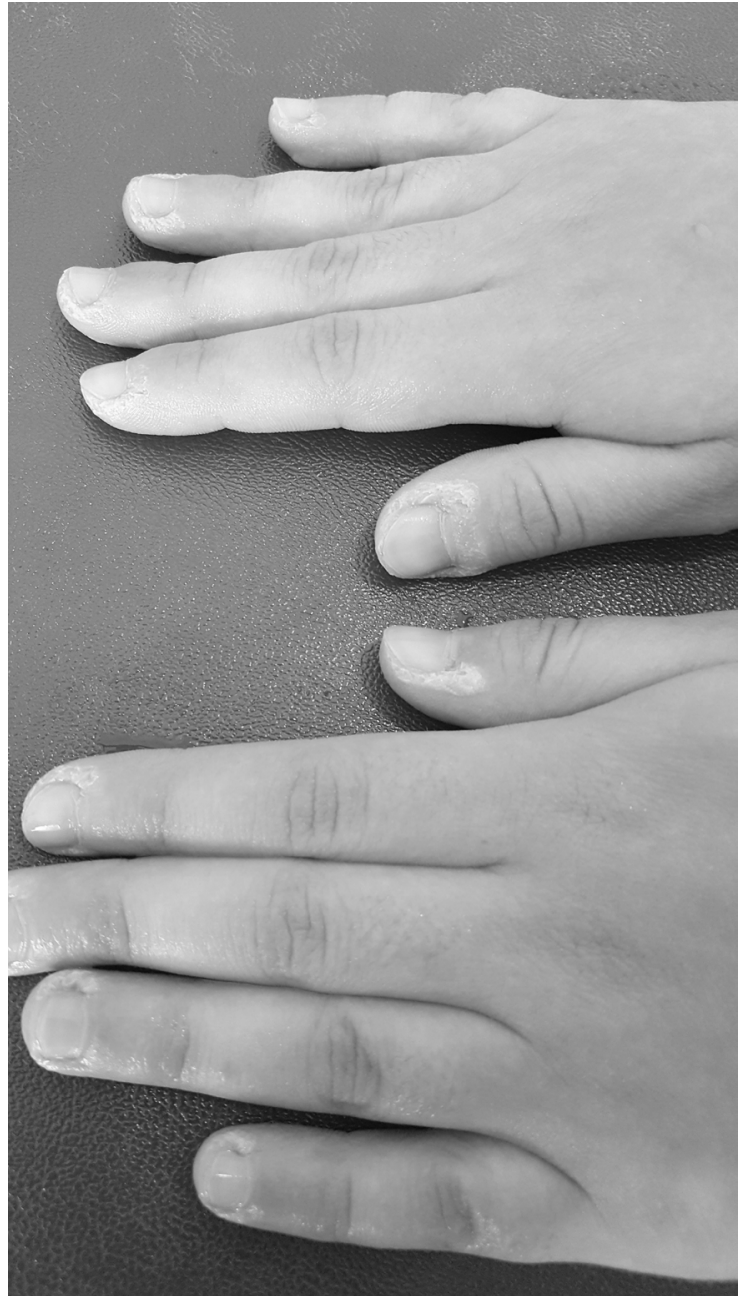

Fig. 1. Aquagenic syringeal acrokeratoderma. White keratodermic plaques on periungual area

region, there are also several cases with the involvement of dorsum of the hand and sole of the feet. A comprehensive literature search revealed only 5 ASA cases with the involvement of dorsal aspect of hand. ${ }^{5-9}$ Thus, our patient represents a rare case of ASA with the involvement of periungual region of the fingers.

Although the pathogenesis is not clear, there are several theories suggesting that the stratum corneum and ecrine ductus and glands play a role in this disease. ${ }^{3}$ It has been suggested that abnormalities of the sweat glands, hyperkeratosis and impairment in the barrier function of the stratum corneum increase the water absorption and lead to the swelling of stratum corneum.

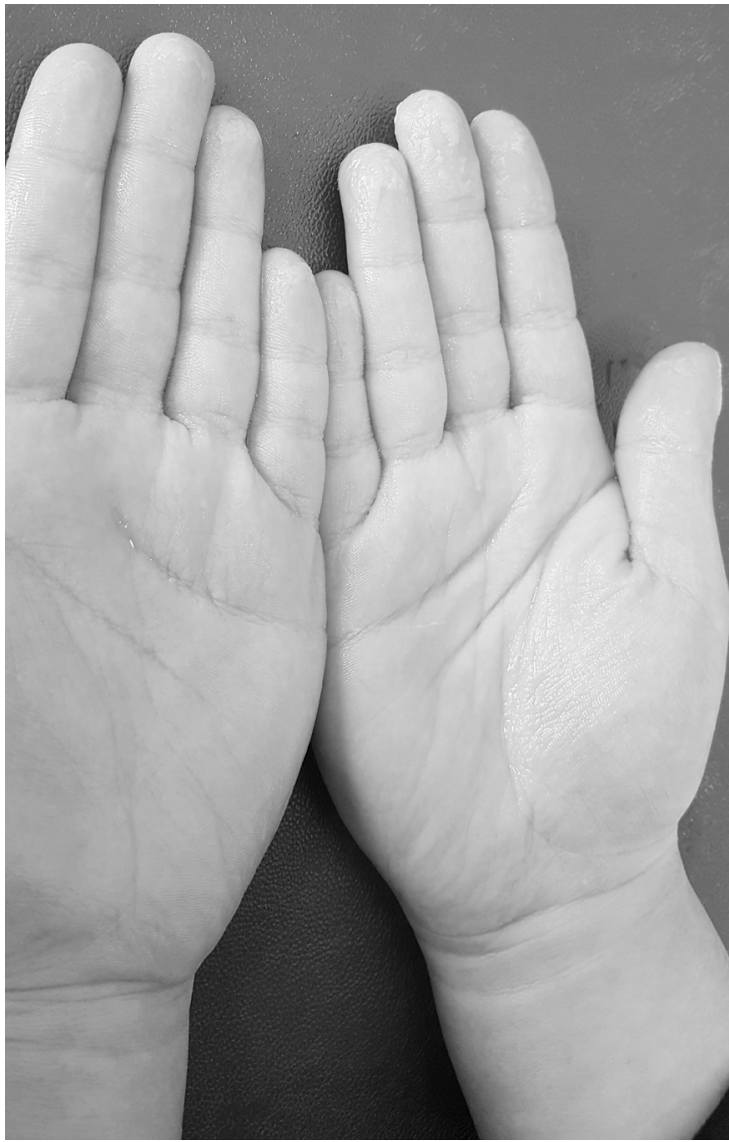

Fig. 2. Aquagenic syringeal acrokeratoderma. White keratodermic plaques on flexor side of distal phalanxes.

This condition has a genetic basis with both autosomal dominant and recessive inheritance. ${ }^{10}$ Familial cases have also been reported in the literature. ${ }^{11}$ ASA is seen in $40-80 \%$ of the

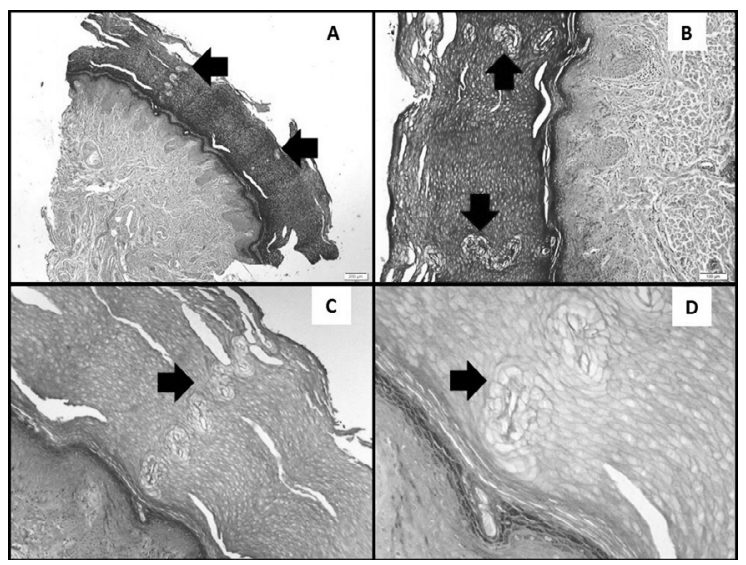

Fig. 3. (A-D) Microscopic photos of the lesion illustrating the intraepidermal ecrin ductus (acrosyringium) dilatation and tortuosity (arrows) (Hematoxylin-eosin stain; original magnification $\times 40, \times 100, \times 200$, and $\times 400$, respectively). 
patients with cystic fibrosis, while the rate is approximately $25 \%$ in the case of being a carrier. $^{12}$ In cystic fibrosis patients, defective chlorine channels create an osmotic gradient and hypertonic sweat secretion results in the dilatation of eccrine channels. ${ }^{13}$ Further investigation was not performed because there was no finding compatible with cystic fibrosis in our patient's evaluation.

In the literature, there are a number of cases secondary to the use of selective cyclooxygenase-2 (COX-2) inhibitors and aspirin. ${ }^{14-16}$ COX-2 inhibitors increase the sodium retention in epidermal keratinocytes resulting in an increase in the water retention capacity of stratum corneum. Belli et al. ${ }^{17}$ presented a case of ASA secondary to ibuprofen use and have suggested that COX inhibition may play a key role in the pathogenesis of ASA and that common use of NSAIDs may increase frequency of the disease. Our patient had never received COX-2 inhibitors.

Yan et al. ${ }^{2}$ have defined the lesions becoming evident by immersing hands of 3 patients in water as "hand in the bucket" sign which has been suggested to be an important finding for the diagnosis of ASA. Clinical and histopathological findings are often sufficient for diagnosis. Sezer et al. ${ }^{18}$ reported that sweat gland openings in the dermatoscopic examination of ASA patients were three times wider than in normal skin areas and they recommended that dermatoscopy be used as a rapid and non-invasive diagnostic method prior to histopathological examination. Dermatoscopy would help to avoid unnecessary biopsies in doubtful cases.

Treatment options include aluminum salts, salicylic acid creams, antihistamines, and botulinum toxin injections. ${ }^{4}$ In our patient, topical $20 \%$ aluminum chloride and hexahydrate produced a partial response to treatment.

In conclusion, while ASA is rare, our case presents an extremely rare condition associated with the involvement of periungual region.

\section{REFERENCES}

1. English III JC, Mc Collough ML. Transient reactive papulo translucent acrokeratoderma. J Am Acad Dermatol 1996; 34: 686-687.
2. Yan AC, Aasi SZ, Alms WJ, et al. Aquagenic palmoplantar keratoderma. J Am Acad Dermatol 2001; 44: 696-699.

3. Itin PH, Lautenschlager S. Aquagenic syringeal acrokeratoderma (transient reactive papulotranslucent acrokeratoderma). Dermatology 2002; 204: 8-11.

4. Luo DQ, Li Y, Huang YB, Wu LC, He DY. Aquagenic syringeal acrokeratoderma in an adult man: Case report and review of the literature. Clin Exp Dermatol 2009; 34: e907-e909.

5. Yoon TY, Kim KR, Lee JY, Kim MK. Aquagenic syringeal acrokeratoderma: Unusual prominence on the dorsal aspect of fingers? Br J Dermatol 2008; 159: 486-488.

6. Rongioletti F, Tomasini C, Crovato F, Marchesi L. Aquagenic (pseudo) keratoderma: A clinical series with new pathological insights. Br J Dermatol 2012; 167: 575-582.

7. Xia Q. Aquagenic acrokeratoderma: Case report with no involvement of the palms. Int J Dermatol 2012; 51: 1391-1393.

8. Luo DQ. Aquagenic acrokeratoderma: A case with family history and unusual involvements of the palms and soles, and the dorsum of fingers and toes. J Dermatol 2011; 38: 612-615.

9. Angra D, Angra K, Rodney IJ. Aquagenic palmoplantar keratoderma with dorsal hand involvement in an adolescent female. JAAD Case Rep 2016; 2: 239-240.

10. Dhawan AK, Bisherwal K, Gandhi V, Kawthekar P, Diwaker P. Aquagenic syringeal acrokeratoderma. Indian Dermatol Online J 2016; 7: 327-329.

11. Saray Y, Seçkin D. Familial aquagenic acrokeratoderma: Case reports and review of the literature. Int J Dermatol 2005; 44: 906-909.

12. Kent JB, Statuta SM, Greer KE, MacKnight JM Watersport hands. Sports Health 2014; 6: 360-362.

13. Baquerizo K, Amini S, Keri JE, Badiavas EV, Elgart GW. Atypical form of transient reactive papulotranslucent acrokeratoderma in a cystic fibrosis carrier. J Cutan Pathol 2013; 40: 413-418.

14. Vildósola S, Ugalde A. Celecoxib-induced aquagenic keratoderma. Actas Dermosifiliogr 2005; 96: 537-539.

15. Khuu PT, Duncan KO, Kwan A, Hoyme HE, Bruckner AL. Unilateral aquagenic wrinkling of the palms associated with aspirin intake. Arch Dermatol 2006; 142: 1661-1662.

16. Carder KR, Weston WL. Rofecoxib-induced instant aquagenic wrinkling of the palms. Pediatr Dermatol 2002; 19: 353-355.

17. Akin Belli A, Dogan G. Role of cyclooxygenase inhibitors in aquagenic syringeal acrokeratoderma. J Eur Acad Dermatol Venereol 2016; 30: e220-e221.

18. Sezer E, Erkek E, Duman D, Sahin S, Cetin E. Dermatoscopy as an adjunctive diagnostic tool in aquagenic syringeal acrokeratoderma. Dermatology 2012; 225: 97-99. 